\title{
Experimental identification of a lattice model for woven fabrics: application to electronic textile
}

\author{
L.A.A. Beex, C.W. Verberne, R.H.J. Peerlings* \\ Department of Mechanical Engineering, Eindhoven University of Technology, P.O. Box \\ 513, 5600 MB Eindhoven, The Netherlands
}

\begin{abstract}
Lattice models employing trusses and beams are suitable to investigate the mechanical behavior of woven fabrics. The discrete features of the mesostructures of woven fabrics are naturally incorporated by the discrete elements of lattice models. In this paper, a lattice model for woven materials is adopted which consists of a network of trusses in warp and weft direction, which represent the response of the yarns. Additional diagonal trusses are included that provide a resistance against relative rotation of the yarns. The parameters of these families of discrete elements can be separately identified from tensile experiments in three in-plane directions which correspond with the orientations of the discrete elements. The lattice model and the identification approach are applied to electronic textile. This is a fabric in which conductive wires are incorporated to allow the embedment of electronic components such as light-emitting diodes. The model parameters are established based on tensile tests on samples of the electronic textile. A comparison between the experimental results of an out-of-plane punch test and the simulation results shows that the lattice model and its characterization procedure are accurate until extensive biaxial tensile deformation occurs.
\end{abstract}

Key words: lattice model, textile, fabric, electronic textile, experimental characterization, experimental mechanics, plasticity

\footnotetext{
*Corresponding author

Address: R.H.J. Peerlings, Department of Mechanical Engineering, Eindhoven University of Technology, P.O. Box 513, 5600 MB Eindhoven, The Netherlands.

Email: r.h.j.peerlings@tue.nl
} 


\section{Introduction}

Woven materials are frequently used, for instance in clothing, bullet-proof armor and reinforced polymeric and ceramic materials. A relatively new application is electronic textile (Edmison et al., 2002; Dalton et al., 2003; Marlescu et al., 2003). Electronic textiles are textiles which contain electronic components such as light-emitting-diodes, sensors, switches, etcetera. The woven fabric acts as a compliant substrate for the electronic components and conductive wires are woven into it in order to electrically connect the individual electronic components. These conductive wires and the connections of the conductive wires with the electronic components must stay intact during manufacturing and use, since failure of the wires and connections entails a malfunctioning product. Mechanical models can be used to study the mechanical interplay between the different constituents of electronic textile.

To model the mechanical behavior of woven materials different approaches can be used. It can for instance be investigated by performing finite element simulations on a single unit cell in which the yarns are discretized in a detailed manner so that, amongst others, yarn-to-yarn interactions are incorporated (Lomov and Verpoest, 2006; Lomov et al., 2007; Badel et al., 2007; Potluri and Sagar, 2008). A limitation of these detailed simulations is their computational cost, which prohibits large-scale simulations.

On the other hand, continuum models are often used for large-scale simulations of woven materials (King et al., 2005; Apedo et al., 2010). They are suitable for large-scale problems because the discrete yarns are not taken into account individually but only in an average sense. A disadvantage of continuum models for woven materials is their inability to capture local (discrete) events such as yarn failure and sliding of yarns. This is an important drawback for the study of electronic textile because the conductive wires are individual, small but relevant features. Other disadvantages are the relatively complex incorporation of large rotations (Peng and Cao, 2005) and the occurrence of numerical difficulties such as locking (Ten Thije and Akkerman, 2008).

Lattice models that employ trusses or beams offer a more natural, intermediate description for woven materials. The discrete members of the mesostructure of these materials are represented by discrete elements such as trusses or beams in these models (Kato et al., 1997; Sharma and Sutcliffe, 2004; Ben Boubaker et al., 2007). An example of a lattice model for a woven fabric is shown in Fig. 1, superimposed on an image of a textile. Individual 
yarn segments are modeled by a discrete element such as a spring. At the yarn-to-yarn contacts, the discrete elements are connected to each other by nodes. The diagonal elements provide the lattice with shear stiffness. In this way the shear stiffness of the fabric, that comes into play if the yarns rotate relative to each other, can be modeled. Local events such as slip in the member-to-member interaction (Ben Boubaker et al., 2007; Liu et al., 2010) and failure of individual members can be taken into account in a natural manner in lattice models (Liu et al., 2010), whereas they are complex to include in continuum models. The discrete conductive wires in electronic textile can also be modelled individually in lattice models, whereas this is not trivially established in continuum descriptions. Furthermore, the high computational cost of detailed sub-yarn models is avoided. An overview of several lattice models is given by Ostoja-Starzewski (2002).

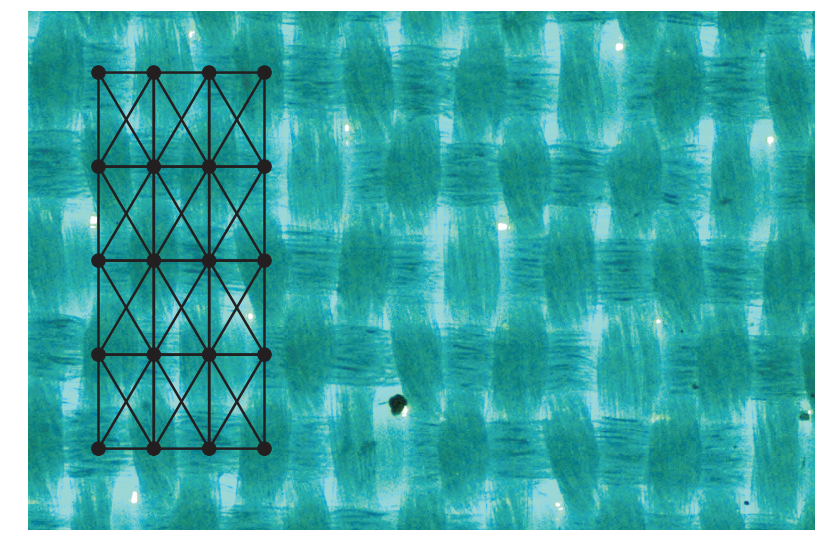

Figure 1: A woven fabric (blue) with 12 unit cells of a lattice model superimposed on it (black). The black lines represent springs or beams which are fixed to each other at the nodes (black dots).

Large-scale lattice computations may still be the computational costly. To overcome this, unit cells of lattice models often represent several unit cells of the woven material, i.e. one truss or beam represents several parallel yarns (Sharma and Sutcliffe, 2004). In some studies (Boisse et al., 2001, 2006; Hamila and Boisse, 2008) the response of the lattice model is translated to the response of a finite element that is also used to represent a number of unit cells. Local events such as element failure can no longer be incorporated in these approaches, but they can still easily deal with large rotations (Sharma and Sutcliffe, 2004) and locking (Sharma and Sutcliffe, 2004; Hamila and 
Boisse, 2008). Also a number of multiscale approaches can be used to increase the efficiency of large-scale computations (Nilakantan et al., 2010; Ha-Minh et al., 2011; Beex et al., 2011).

Identification approaches to establish the parameters of the different discrete elements in lattice models can be complex since the discrete elements are all mechanically connected. Consequently, they influence each other during the experimental parameter identification. Identification approaches can therefore be somewhat elaborate (Sharma et al., 2003; Sharma and Sutcliffe, 2004; Boisse et al., 2006). In this paper a rather general two-dimensional lattice model for woven materials is proposed that can be characterized in a straightforward manner. From three types of in-plane tensile tests that are performed in the orientations of the three families of discrete elements, the parameters of the discrete elements are individually established. In this way no (complex) inverse problem has to be solved to establish the material parameters.

In order to separately identify the discrete elements, the mutual influence must be negligible. To this end, the compressive responses of all elements in the lattice model proposed in this paper vanish. The lattice model and its identification procedure are applied to a woven electronic textile including conductive wires, but it can be used for any woven material that is characterized by a compliant rotational stiffness relative to the axial stiffness, such as e.g. metal grids to reinforce concrete (Han and Tsai, 2011).

The outline of this paper is as follows. First the electronic textile is described and the in-plane experiments on the electronic textile are discussed. Also the fabric strains at which the conductive wires fail are identified. Subsequently, the lattice model is detailed and the identification procedure is discussed. In section 5 the lattice model including the identification procedure is validated by a three-dimensional punch test. Overall experimental and predicted deformations are compared as well as the experimental and predicted punch force-punch displacement curves; failure of the conductive wires is also evaluated. Finally, conclusions are presented.

\section{In-plane experiments}

The fabric considered here is an electronic textile produced by TiTV (www.titv-greiz.de). It is a densely woven fabric with embedded conductive wires (see Fig. 2). The conductive wires are predominantly oriented in warp 
direction and on average one wire is present on 65 warp yarns. In weft direction an insignificant number of conductive wires are present. The conductive wires consist of a number of copper filaments (see Fig. 2). At regular intervals they have some clearance with respect to the textile to allow the mounting of electronic components (see Fig. 2). The textile yarns of the fabric contain different fibers of dtex 76. The yarns in warp direction are turned 600 times per meter and those in weft direction are turned 120 times per meter. The density of the warp and weft yarns is $11000 \mathrm{~m}^{-1}$ and $8900 \mathrm{~m}^{-1}$ respectively. The warp and weft yarns are woven in a three layer pattern.
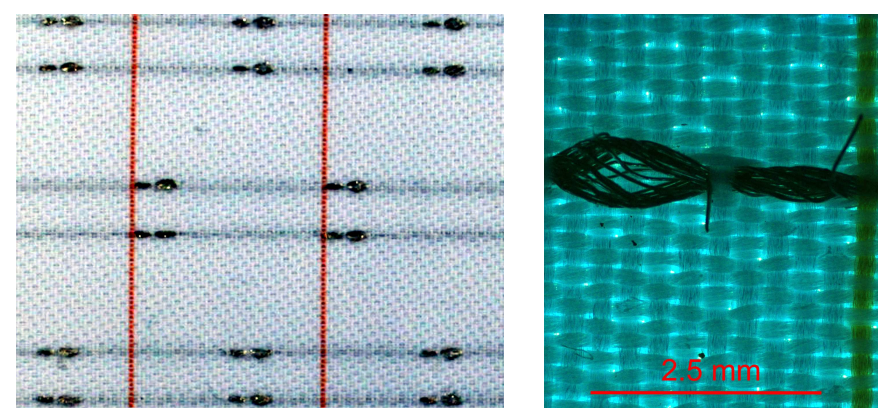

Figure 2: (Left) the electronic textile with the warp direction in horizontal direction and (right) a microscopic image of the electronic textile. The conductive wires are mainly oriented in warp direction. The clearance of the conductive wires is clearly visible.

\subsection{Methodology}

Tensile test samples of the electronic textile (including the conductive wires) of $100 \times 29 \mathrm{~mm}^{2}$ are taken in three directions; in warp and weft direction and at an angle of $45^{\circ}$ with respect to the warp direction. The tensile tests in the latter direction corresponds to the bias extension test (Sharma et al., 2003; Peng and Cao, 2005). The nominal thickness of the samples is measured as $0.35 \mathrm{~mm}$, although this thickness is somewhat arbitrary since the samples are highly heterogeneous. The samples are fixed in between two clamps with a rough surface together with one piece of double-sided tape to increase the fixation. The gauge length of all samples is approximately 60 $\mathrm{mm}$. The used tensile tester (Instrom 5566) has a load cell of $500 \mathrm{~N}$. The strain rate in the experiments in warp and weft direction is $1.67 \cdot 10^{-3} s^{-1}$ and in diagonal direction $3.33 \cdot 10^{-3} \mathrm{~s}^{-1}$, in order to keep the strain rates 
of the individual yarns as similar as possible. No influence of time on the material response is investigated.

During the experiments, images of the strained samples are recorded, to which an optical strain measurement technique is applied to determine the local strains. Undesired effects such as slip in the clamps and deformation of the load cell are therefore circumvented in the strain measurement. Furthermore, in the tensile test in diagonal direction (bias extension test), the pure shear strains that only occur in region $\mathrm{C}$ in Fig. 3, as is well described in literature (Sharma et al., 2003; Peng and Cao, 2005; Ten Thije and Akkerman, 2008), can be established without any influence of the constraining influence of the clamps (in regions $\mathrm{A}$ and $\mathrm{B}$ ). To determine the engineering stress of the samples the measured cell force and the original nominal cross-sectional area are used.

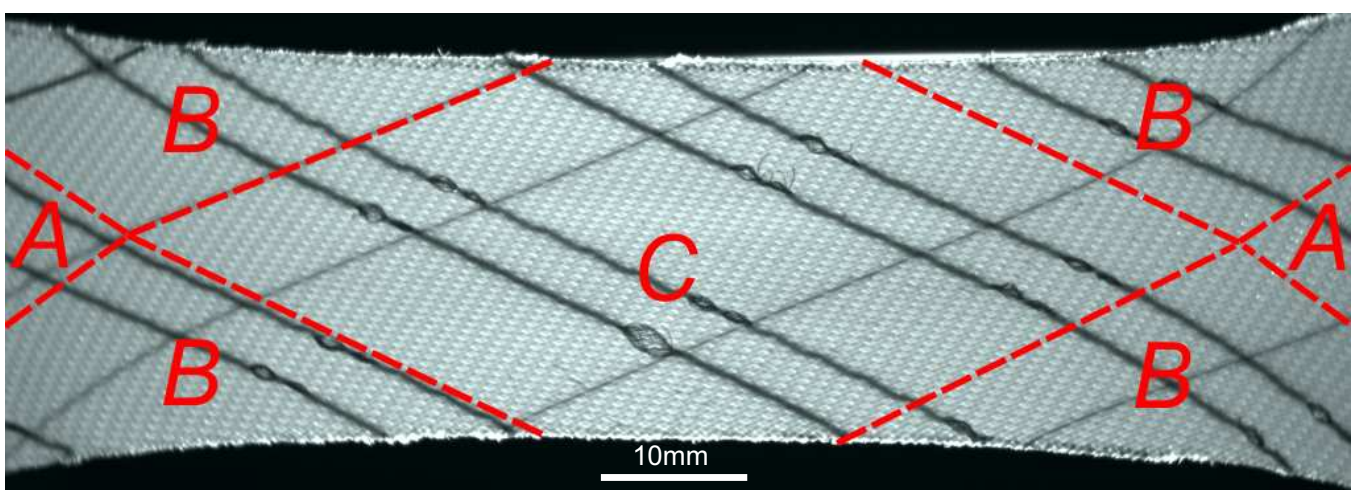

Figure 3: Three deformation modes (A, B and C) occur in the samples during the bias extension tests due to the influence of clamping. The conductive wires are shown in black while the red yarns (shown in grey) correspond to regular weft yarns as in Fig. 2.

To investigate the failure of the conductive wires within the fabric, $\mathrm{X}$-ray images are made (Phoenix PCB analyzer, using $60 \mathrm{kV}$ and $20 \mu \mathrm{m}$ ) after the tensile tests in warp direction. Although these images are not direct input for the experimental identification, they are used in section 5 to evaluate the lattice model and the identification procedure.

\subsection{In-plane stress-strain responses}

The engineering-stress/engineering-strain responses of the in-plane tensile experiments are shown in Fig. 4. The engineering stress and engineering 
strain are used here, to obtain a first impression of the textile behaviour in the different directions. In section 4 however, the true stress and true strain of the individual truss elements are determined based on the engineering stress and engineering strain of the textile, since the software in which the lattice model has been implemented uses the true-stress/true-strain constitutive relations. To calculate the engineering stress and engineering strain shown in Fig. 4, the force measured by the load cell is divided by the nominal cross-sectional area and the measured clamp displacement is divided by the undeformed clamped length of the textile samples. Only one response is shown in each direction; the experimental scatter of each response is relatively small (Verberne, 2011).

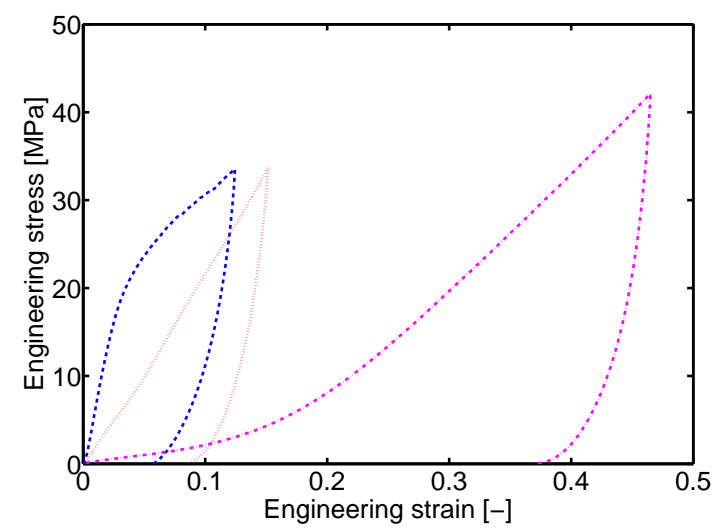

Figure 4: Engineering stress-engineering strain responses of the electronic textile in warp (blue, dashed), weft (red, dotted) and diagonal direction (magenta, dashed-dotted).

The responses in warp and weft direction show similar levels of stress for the same applied strain level. However, the shapes of the curves are clearly different from each other (see Fig. 4). The warp response shows a nonlinear loading behavior whereas the loading behavior of the weft direction is virtually linear. Tensile tests on single yarns (not shown) have indicated that this different behavior in the two directions is caused by the fact that the two types of yarns are different: they are both made from the same material but have a different number of turns per meter. All unloading responses show that a large amount of inelastic deformation has occurred during the tensile tests.

The diagonal direction exhibits an initially extremely compliant response, which increases at a strain of approximately $14 \%$ (see Fig. 4). This compli- 
ant shear behavior is typical for woven materials and also occurs for instance in woven grids to reinforce concrete (Han and Tsai, 2011). The response is determined by the rotation of the warp and weft yarns relative to each other. Initially, this rotation solely experiences friction in the yarn-to-yarn. However, at higher levels of strain, and thus larger rotations, the warp and weft yarns start to make contact with each other, which leads to an increasingly stiffer response. In the densely woven fabric considered here this effect occurs at moderate strains, but for less densely woven fabrics it may occur much later and the nonlinear response is thus more pronounced (Sharma and Sutcliffe, 2004).

\subsection{Failure of the conductive wires}

X-ray images of the electronic textile samples after the tensile experiments in warp direction are presented in Fig. 5. At the location where the conductive wires have some clearance, the copper filaments in each wire can be distinguished. Plastic deformation and failure of the conductive wires can only be observed at the clearances.

For the undeformed sample and the samples strained to $2 \%$ and $6 \%$ (engineering strain), no failure of the wires can be seen. Although the sample that is strained to $6 \%$ clearly shows plastic deformation in the wires, the wires are still intact and their conductivity is unaffected.

Failure of the wires starts at a strain of approximately $7 \%$, as becomes clear from image D in Fig. 5. A number of copper filaments in the conductive wires are broken at the clearance of the wires. For larger strains the number of broken filaments increases and in some cases all filaments of a conductive wire are broken, so that no electrical contact is made anymore.

\section{Lattice model}

A unit cell of the proposed two-dimensional lattice model for the electronic fabric is shown in Fig. 6. The tow truss elements represent warp and weft yarn segments from one yarn-yarn crossing to the location of the next one. The unit cell's dimensions match the dimensions of the unit cell of the 

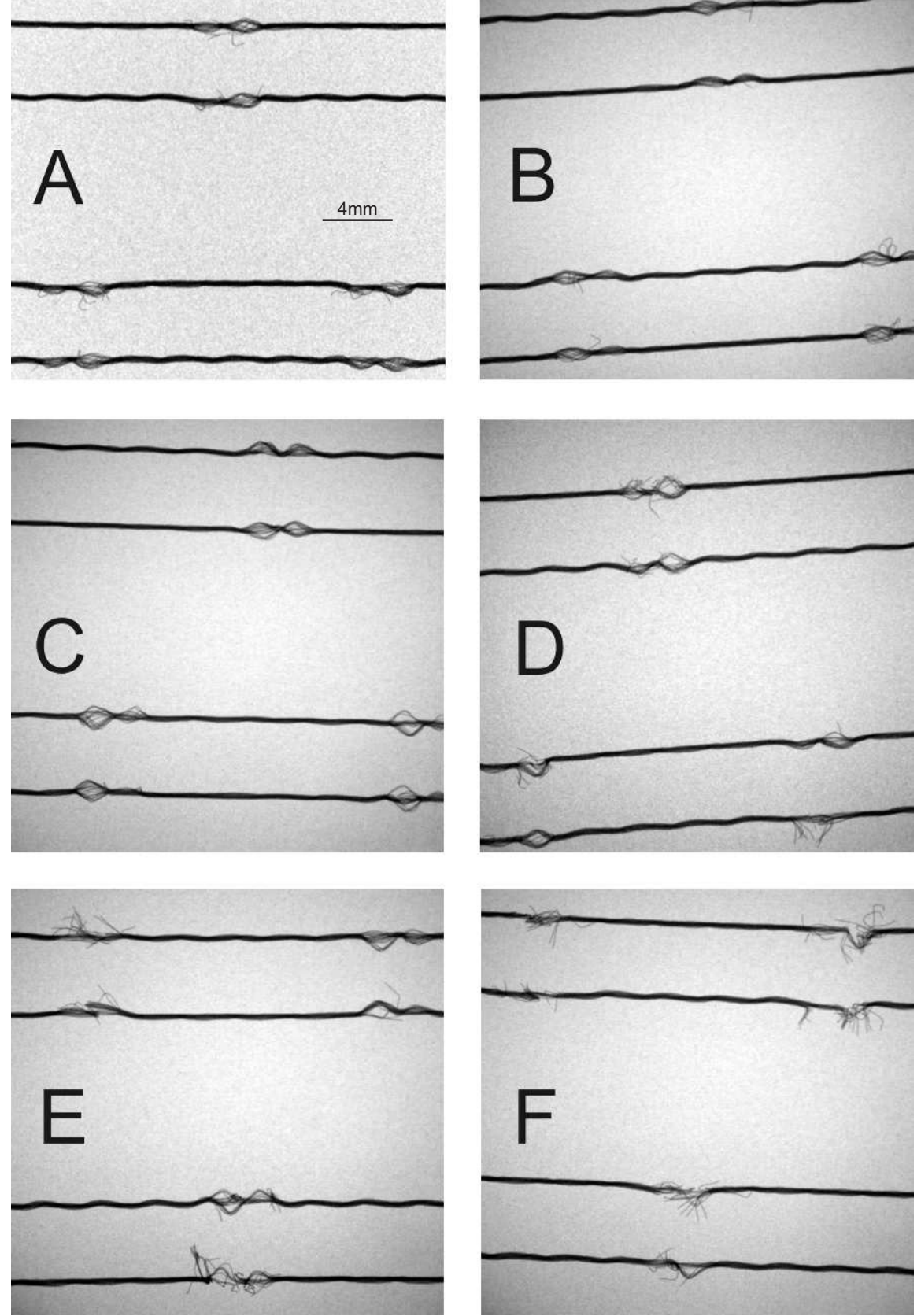

Figure 5: X-ray images of an undeformed sample (A) and after 2\% (B), 6\% (C), 7\% (D), $8 \%(\mathrm{E})$ and $9 \%(\mathrm{~F})$ straining in warp direction. The conductive wires can be distinguished but not the woven fabric. 
discrete mesostructure of the fabric, i.e. each yarn is represented explicitly by a (chain of) truss(es).

The diagonal trusses provide the unit cell with shear stiffness, in correspondence with the lattice model of Sharma and Sutcliffe (2004), except that two diagonal elements are used instead of one. The advantage of using two diagonal elements per unit cell is that uniaxial deformation in warp and weft direction can be described at the scale of a single unit cell. In contrast to the lattice model of Kawabata et al. (1973), out-of-plane phenomena such as out-of-plane contraction and undulation are not specifically modeled, but the influence of many out-of-plane mechanisms on the in-plane responses are incorporated in the material descriptions of the truss elements. The out-ofplane bending stiffness is not captured however, but this is rather compliant. Furthermore, no conductive wires are individually modeled in the lattice model since they hardly contribute to the response (Verberne, 2011) due to their small number (one conductive wire is present on 65 warp yarns).

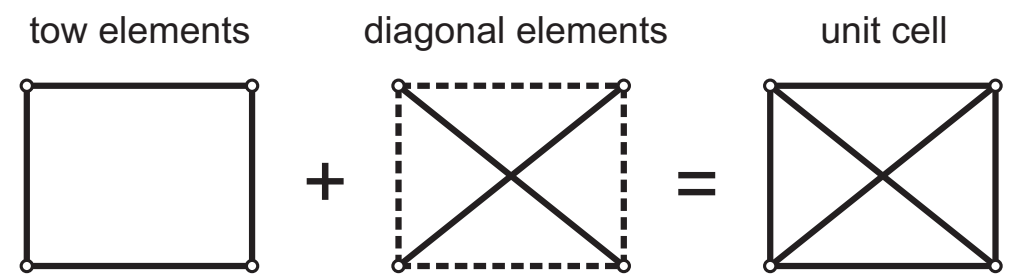

Figure 6: Four tow elements, representing the yarns (left), and two diagonal elements (center), providing rotational stiffness, are used in a rectangular unit cell of the lattice model (right).

In the lattice model the (discrete) yarn segments, represented by the tow truss elements, carry no force when they are compressed. The reason for this is that it is assumed that they buckle as soon as they are loaded in compression. Also the diagonal truss elements are considered to carry no force in compression. As a result, the simple shear loading only charges one diagonal truss element while the other one is compressed without axial stress (see ahead to the right image in Fig. 8).

Since the Hencky (i.e. true) strain is used in the numerical implementation (in MSC.Marc), the axial strains of the individual trusses are expressed in terms of it: 


$$
\epsilon=\ln (\lambda)
$$

where $\lambda=l / l_{0}$ is the axial stretch factor, with $l$ and $l_{0}$ the current and initial length respectively. Since inelastic deformation occurs in the stress-strain responses of Fig. 4, an elastoplastic model is adopted for the trusses. The axial strain can be split in an elastic and plastic part as follows:

$$
\epsilon=\epsilon_{e}+\epsilon_{p}
$$

where $\epsilon_{e}$ is the axial elastic Hencky strain and $\epsilon_{p}$ the axial plastic Hencky strain.

The elastic response in each truss is governed by Hooke's law as follows:

$$
\sigma=E \epsilon_{e}
$$

where $\sigma$ represents the axial true stress and $E$ is the Young's modulus of the material.

The lateral contraction due to elastic straining is neglected. The plastic deformation, on the other hand, is assumed to be incompressible. The true stress in a truss can therefore be determined from the engineering stress via the following expression:

$$
\sigma=\sigma_{e n g} \lambda_{p}
$$

where $\sigma_{e n g}$ is the axial engineering stress and $\lambda_{p}=\exp \left(\epsilon_{p}\right)$ is the axial plastic elongation factor.

Because the typical nonlinear responses in the different directions in Fig. 4 show that the material behaves plastically from the very beginning of loading, the loading response of the trusses is described by the plastic hardening. The elastic part of the constitutive model is used to describe the unloading response. To this end, a low initial yield stress, $\sigma_{y 0}$, is used and the hardening law is progressive. This is schematically shown in Fig. 7.

At this point the precise hardening law is not yet formulated since the most suitable hardening law appears out of the identification procedure. For 


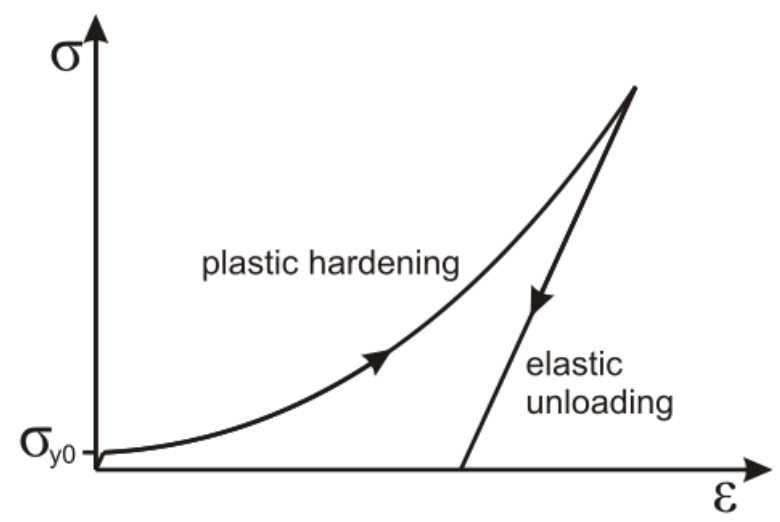

Figure 7: Schematic illustration of the uniaxial stress-strain response of the material description used for the trusses. The initial yield stress is indicated by $\sigma_{y 0}$.

this reason, the current yield stress $\sigma_{y}$ of the three types of truss elements remains a yet to be defined function of the equivalent plastic strain $\bar{\epsilon}_{p}$, i.e. $\sigma_{y}\left(\bar{\epsilon}_{p}\right)$.

The lattice model is implemented in the software package MSC.Marc. The implementation uses an updated Lagrange approach to deal with large deformations and rotations. The current local axes and cross-sectional area of the truss elements are updated every iteration. The Mohr-Coulomb criterion is used to distinguish between tension and compression; its parameters are selected such that in compression the responses of the truss are negligible.

\section{Identification procedure}

Considering uniaxial loading in warp and weft direction for a single unit cell (see the left and center image in Fig. 8), it can be observed that only the discrete elements that are oriented in the loading direction contribute to the mechanical response. The reason for this is that the shear response (modeled by the diagonal elements) is compliant (the magenta curve in Fig. 4) compared to the response in warp and weft direction (the other two curves in Fig. 4). The diagonal elements may thus be expected to have a comparatively low stiffness. As a result only the elements oriented in the loading direction contribute to mechanical response during warp and weft loading. Note that, although the stiffness of the diagonal elements increases for strains larger 
than $14 \%$ (see Fig. 4), this strain is not exceeded since the warp and weft strains in Fig. 4 remain below $14 \%$.

On the other hand, for the bias extension test (see the right image in Fig. 8), only the diagonal element that is oriented in the loading direction contributes to the mechanical response. The reason for this is that the four stiffer elements that represent yarn segments act as a mechanism. The diagonal element that is not oriented in the loading direction is compressed without stress, since no resistance against compression is included in the lattice model.

During the three in-plane tests discussed in section 2 , it is thus reasonable to assume that only the elements oriented in the respective loading directions contribute to the mechanical response. Hence, a full uncoupling between tension in warp direction, tension in weft direction and tension in diagonal direction is assumed. The conditions for this assumption to hold are, as mentioned above, that the intrinsic material behavior of the fabric shows a compliant shear response compared to the in-plane principal directions and that the elements under compression show no stress. The results from the three in-plane tests in Fig. 4 can now directly be used to determine the parameters of the three families of discrete elements associated with the three directions. Below it will be explained how the parameters of each family of elements can be established based on these tensile test results.

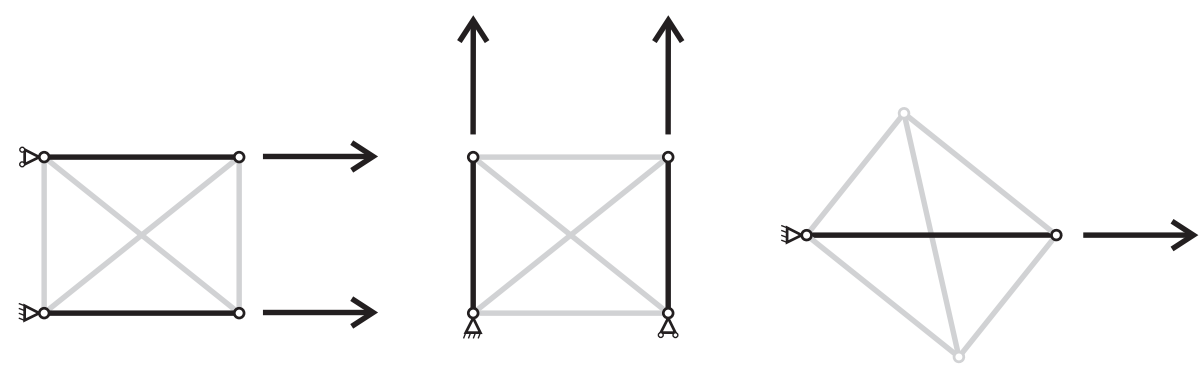

Figure 8: Schematic representation of three in-plane loading situations for the identification procedure in which only the truss elements oriented in the loading direction contribute to the response (black). The other truss elements (grey) are inactive or contribute negligibly. The left image represents loading in warp direction, the center image loading in weft direction and the right image diagonal loading.

Although the diagonal truss elements are oriented at an angle of $29^{\circ}$ with respect to the warp elements and the diagonal tensile tests (bias extension 
tests) are performed at an angle of $45^{\circ}$ to the warp direction, the stress-strain responses from the bias extensions tests are directly used for the identification of the parameters of the diagonal trusses. Clearly, this difference in angle is not optimal, but the predicted unit cell responses nevertheless match the experimental responses well (see ahead to Fig. 12).

\subsection{From engineering stress to element stress}

Before the material parameters of the different families of truss elements can be established, the geometric parameters are set. The nominal initial area $A_{0}$ of all trusses is set to $0.0155 \mathrm{~mm}^{2}$. This value is in the order of magnitude of the actual yarns. In principle, since only the force transmitted by the trusses matters, any diameter can be selected as long as it is dealt with in a consistent manner. The length of the elements $l_{0}$ is based on the microscopic images of Fig. 2. The geometric parameters are presented in Table 1.

Before the parameters of the discrete members can be fitted, first the engineering stresses obtained from the tensile tests, $\sigma_{\text {eng,t }}$ (see Fig. 4 ), must be converted to the engineering stresses of the individual discrete elements, $\sigma_{\text {eng }}$. The reason is that the engineering stresses obtained from the tensile tests, $\sigma_{\text {eng,t }}$ (see Fig. 4), are computed as if the electronic textile is a continuum, whereas the engineering stresses of the discrete members are needed (see Fig. 9). Therefore, the ratio between the yarn area, $A_{0}$, and the nominal cross-sectional area of the textile, $A_{n}$, must be taken into account as follows:

$$
\sigma_{e n g}=\frac{\sigma_{e n g, t} A_{n}}{A_{0}}
$$

where $A_{n}$ is the nominal area associated with a single discrete element (see Fig. 9).

The areas are determined based on the in-plane dimensions of the unit cell (see Fig. 2), the dimensions of the yarns and the thickness of the electronic textile; they can be found in Table 1.

\subsection{Elastic behavior}

Now that the engineering stresses of the elements can be determined, the three Young's moduli can be fitted. As mentioned before, the elastic part of the constitutive model is used to describe the three unloading responses. One has to take into account that at the moment that unloading takes place, 


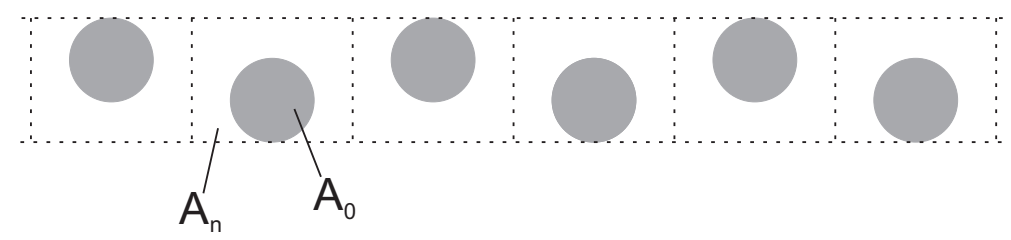

Figure 9: A schematic representation of a cross section of the fabric in out-of-plane direction. The (initial) area of an element is represented by $A_{0}$ and the nominal area associated with it by $A_{n}$.

the cross-sectional area is deformed, since during loading plastic deformation occurs in an incompressible manner. The true stress at the moment of unloading must thus be employed to fit the Young's moduli. To determine this true stress, it is assumed that all strain applied until the point of unloading is plastic strain and $\lambda_{p}$ in Eq. (4) may thus be replaced by $\lambda$. The Young's moduli are fitted on the highest $40 \%$ (in terms of stress) of the unloading responses. Damage has not taken place at this point, since cyclic loading responses show that the unloading stiffness remains similar (Verberne, 2011). The resulting curves and the fits of the moduli are shown in Fig. 10. The values of the moduli are given in Table 1 .

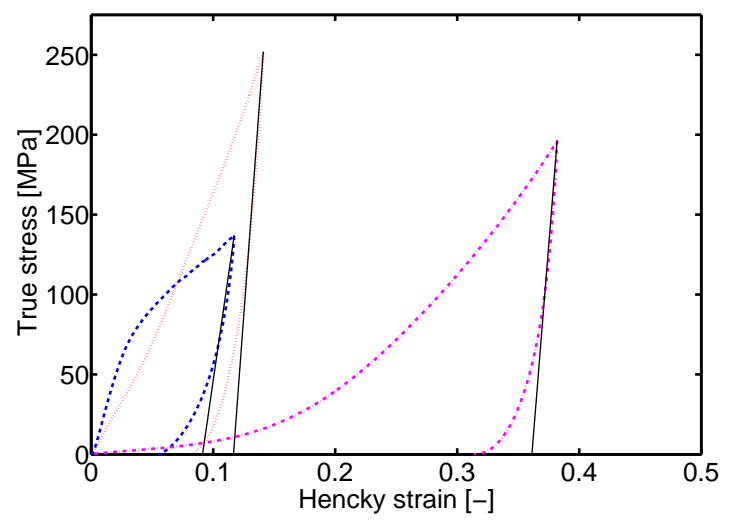

Figure 10: The true stress as a function of the total Hencky strain of the individual elements in warp (blue, dashed), weft (red, dotted) and diagonal direction (magenta, dashed-dotted) and the corresponding fits of the Young's moduli (black, solid). 


\subsection{Plastic behavior}

To ensure that the plastic part of the constitutive model of the elements is used for the entire loading responses, small yield stresses are used for all three families of elements (see Table 1). A lower value than $0.2 \mathrm{MPa}$ is theoretically desired, but smaller values lead to convergence problems in the final validation simulation as described in Section 5. Furthermore, this yield stress is sufficiently small for accurate fits (see ahead to Fig. 12).

To determine which hardening law can be used and to fit its parameters, the true stress-equivalent plastic strain curves are presented in a log-log diagram in Fig. 11. The (effective) plastic strain has been determined by subtracting, at each level of stress, the elastic strain as given by the Young's moduli determined above from the total strain. Consequently, the Young's moduli have no influence on the plastic hardening behaviour that is used to describe the entire loading curves. The following power law seems suitable for the hardening behavior of the three responses, since the log-log diagrams are more or less linear in the regimes of influence:

$$
\sigma_{y}=\sigma_{y 0}+H\left(\bar{\epsilon}_{p}\right)^{n}
$$

where $\sigma_{y 0} \approx 0$ is the initial yield stress and $H$ and $n$ are hardening parameters. The resulting fits of the hardening behavior and the corresponding parameters are shown in Fig. 11 and Table 1 respectively.

Table 1: Established parameters of the three families of elements.

\begin{tabular}{|l|l|l|l|}
\hline & warp & weft & diagonal \\
\hline$A_{0}\left[\mathrm{~mm}^{2}\right]$ & 0.0155 & 0.0155 & 0.0155 \\
\hline$l_{0}[\mathrm{~mm}]$ & 0.288 & 0.161 & 0.330 \\
\hline$A_{n}\left[\mathrm{~mm}^{2}\right]$ & 0.0563 & 0.1008 & 0.0492 \\
\hline$\sigma_{y 0}[\mathrm{MPa}]$ & 0.2 & 0.2 & 0.2 \\
\hline$E[\mathrm{GPa}]$ & 5.276 & 10.32 & 9.500 \\
\hline$n[-]$ & 0.371 & 1.17 & 2.52 \\
\hline$H[\mathrm{MPa}]$ & 315.4 & 2,816 & 2,372 \\
\hline
\end{tabular}




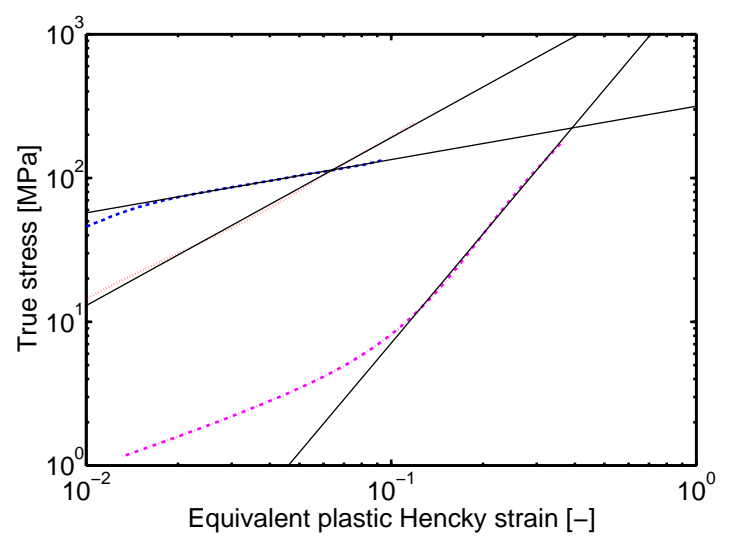

Figure 11: Log-log diagram of the true stress of the discrete elements as a function of the equivalent plastic Hencky strain in warp (blue, dashed), weft (red, dotted) and diagonal direction (magenta, dashed-dotted) and the fits of the hardening behavior (black, solid).

\subsection{Validation of the unit cell response}

The responses of a unit cell of the lattice model in the three tested directions are shown together with the experimental responses in Fig. 12. In the lattice model the linear Mohr-Coulomb criterion is used to make the compressive responses of the individual elements ten times more compliant than the tensile responses. The in-plane stress-strain curves in warp and weft direction as well as the major part of the response in diagonal $\left(45^{\circ}\right)$ direction correspond well with the experimental curves. A small discrepancy can be distinguished in the response in weft direction. This is caused by the formulation of the Mohr-Coulomb criterion in MSC.Marc and the values that are used to ensure a large difference between the tensile response and compressive response.

Only the final part of the diagonal response deviates from the experimental response. A small part of this deviation, between a strain of approximately $28 \%$ and $38 \%$, is caused by the contribution of compressive behavior of the diagonal element that is not oriented in the direction of the loading. At an engineering strain of $38 \%$ (see Fig. 12), all tow elements are oriented in the same direction as the loaded diagonal element and they thus no longer act as a mechanism and start to contribute to the predicted response. As a result, the response of the unit cell increases significantly. This effect is less pronounced in the experiment, in which the transition from relative rotation to a stretching dominated response is more gradual. 


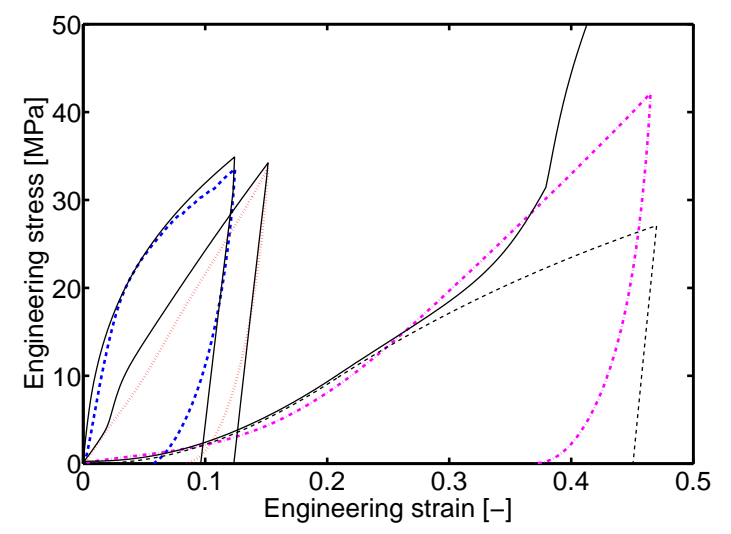

Figure 12: Comparison of the experimentally obtained engineering stress-engineering strain curves of Fig. 4 in warp (blue, dashed), weft (red, dotted) and diagonal direction (magenta, dashed-dotted) and the responses of a unit cell loaded in the same directions (black, solid). The response of a single diagonal truss element loaded in its axial direction (dashed) is also shown for comparison.

The material parameters of the diagonal elements are based on the bias extension test in which the loading angle is $45^{\circ}$ with respect to the warp direction. In the unit cell of the lattice model however, the diagonal elements are oriented at angles of $29^{\circ}$ to the warp direction. To validate the diagonal response of the unit cell, the unit cell is loaded in $45^{\circ}$. Interestingly, the response of a single diagonal element loaded in its axial direction (the black, dashed curve in Fig. 12) shows that there is no significant discrepancy with the response of the unit cell in diagonal $\left(45^{\circ}\right)$ direction. Only from an engineering strain of $28 \%$ onwards the responses start to diverge due to the contribution of the remaining elements of the unit cell. Sliding may also have an influence on this, since it is not incorporated in the model but may occur in bias extension (Lomov et al., 2008). The bias extension test results thus turn out to be rather insensitive to the loading direction.

\section{Simulation of an out-of-plane punch test}

To validate the lattice model, an out-of-plane punch test (see Fig. 13) is simulated and predictions made for it are compared to experimental results. An out-of-plane punch test is used here, because out-of-plane deformations typically occur if electronic textile is used for clothing. The punch test can 
for instance be regarded as a human elbow or knee pressing into a piece of electronic textile. Since broken conductive wires result in malfunctioning electronic textile products, the behaviour of the conductive wires is particularly investigated.

The test setup for this experiment is shown in Fig. 13. In the punch test, a sample of electronic textile with a free area of $100 \times 100 \mathrm{~mm}^{2}$ is fixed between two clamps in warp direction. A sphere with a diameter of $30 \mathrm{~mm}$ is placed below the center of the sample and punches the sample at a velocity of $1 \mathrm{~mm} / \mathrm{s}$. This results in an average strain rate of the warp yarns at the center of the specimen of $8.3 \cdot 10^{-3} \mathrm{~s}^{-1}$, which is of the same order of magnitude as the strain rates used in the tensile tests discussed in section 2.

During the punch test, the reaction force on the punch is measured as a function of its displacement. The tensile tester is equipped with a $10 \mathrm{kN}$ load cell with a stiffness of 16,400 N/mm for this purpose. Since the warp yarns are fixed in the clamps at two edges and the punch is moved by a large distance (50-60 $\mathrm{mm}$ ), large macroscopic and local deformations are expected.

To simulate the punch experiment, only a quarter of the specimen is modeled using symmetry boundary conditions (see Fig. 13). The model consists of $9 \times 16$ unit cells in warp and weft direction respectively (170 lattice nodes). This means that one unit cell in the punch simulation corresponds to $19.5 \times 19.5$ fabric unit cells as described in sections 3 and 4 . To ensure that a unit cell as used in the punch simulation has the same response as $19.5 \times 19.5$ original unit cells, the cross-sectional areas of the truss elements are 19.5 larger than those used for the identification.

The clamps in which the specimen is fixed are modeled by displacement boundary conditions on the edge of the model that is oriented orthogonally to the warp direction. The punch is considered as a frictionless rigid body in the simulation. Since the velocity of the punch is small, a quasistatic analysis can be performed. To ensure that some amount of out-of-plane stiffness is present in the model before the punch makes contact with the lattice, a bilinear initial out-of-plane displacement is given to the lattice, with an amplitude of $1 \mathrm{~mm}$. In the true punch simulation the maximum displacement of $52.5 \mathrm{~mm}$ is reached in 51,500 increments. The convergence tolerance is formulated in terms of the relative displacements and is set to a value of 0.01 . Smaller tolerances lead to the same results. 

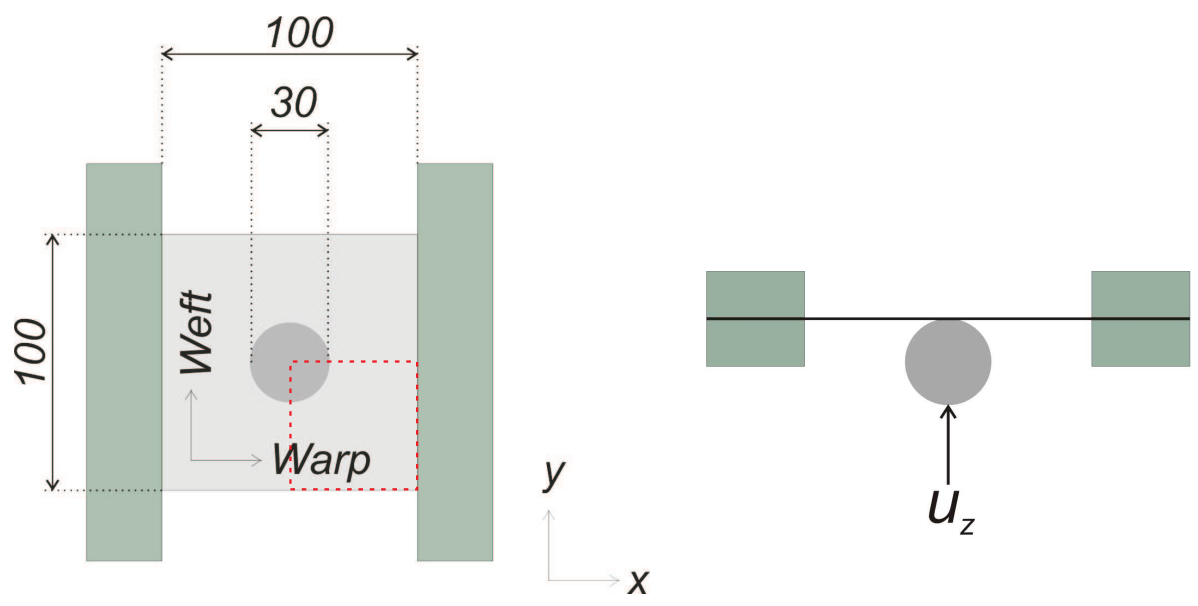

Z

Figure 13: Top view (left) and side view (right) of the test setup for the punch experiment. The clamps are shown in green and the spherical punch in dark grey. The applied punch displacement in the experiments is denoted by $u_{z}$ (right). The quarter of the electronic textile that is modeled in the simulation is indicated by the red dashed square (left). The dimensions are given in $\mathrm{mm}$.

\subsection{Force-displacement response}

The force-displacement curve is presented in Fig. 14 together with four experimental curves. The initial response is compliant since hardly any outof-plane stiffness is present at the start of the test. However, the slope increases rapidly until a punch displacement of $20 \mathrm{~mm}$ is reached. From $20 \mathrm{~mm}$ onwards the slope of all experimental curves remains more or less constant until a displacement of approximately $40 \mathrm{~mm}$ is reached. At this punch displacement already one of the curves has deviated from the average trend of the remaining curves due to slip in the clamps. At a displacement of $40 \mathrm{~mm}$ the second curve starts to deviate due to a large amount of slip in the clamps and at larger displacements this can be observed for the remaining two curves as well. In none of the experiments the electronic textile fails; slip from the clamps determines the force drop in all cases. The deformation of the samples is presented in the left parts of the four images in Fig. 15.

The numerically predicted force-displacement curve presented in Fig. 14 shows a good agreement with the experimental curves until a punch displacement of approximately $25 \mathrm{~mm}$. At this displacement the local axial strains of the warp elements on top of the punch are approximately $14 \%$. In the 


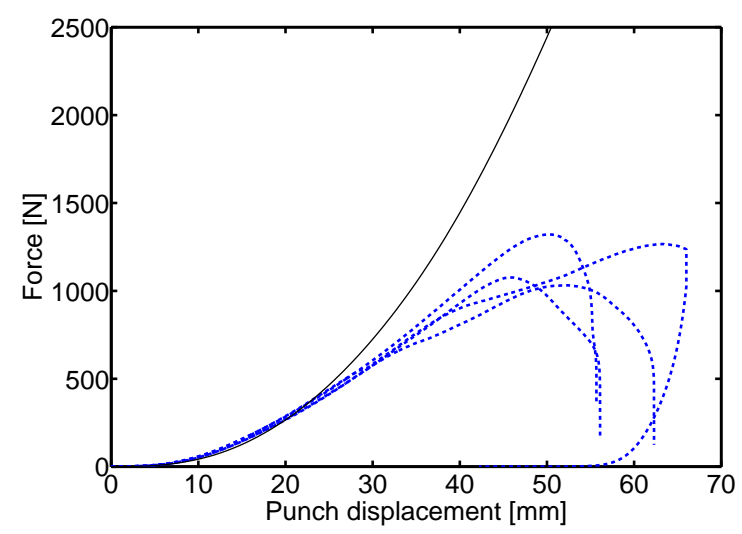

Figure 14: The experimental (blue, dashed) and predicted (black, solid) force as a function of the punch displacement.

warp elements between the punch and the fixed edge of the model local axial strains of 9-10\% are observed. However, towards the free edge of the model, which is parallel to the warp yarns, the local axial warp strains decay to approximately $3 \%$ over only 6 out of 16 unit cells in weft direction.

The good accuracy of the simulation until a punch displacement of 25 $\mathrm{mm}$ can also be observed in images A (at a displacement of $10 \mathrm{~mm}$ ) and B (at a displacement of $20 \mathrm{~mm}$ ) in Fig. 15, since the free edge in the simulations deforms exactly as in the experiment. For larger punch displacements (image $\mathrm{C}$ and D), a disagreement of these free edges can be observed.

From a displacement of approximately $25 \mathrm{~mm}$ onwards, the slope of the computed curve continues to increase, whereas that of the experimentally obtained curves remains constant and then drops. This discrepancy can be related to a number of causes, but the most important one is the poor performance of the unit cell for extensive biaxial deformation. For large biaxial deformations the diagonal truss elements, that are only meant to describe the in-plane shear response of the textile, elongate significantly and start to contribute significantly to the mechanical response of the model. The maximum engineering strain of the diagonal trusses is approximately 0.56 (for comparison with Fig. 12).

Predicted local relative rotation angles between the warp and weft yarns have not been compared to the local experimental shear angles because of the (limited) experimental equipment. Macroscopically, the shear angles seem to 

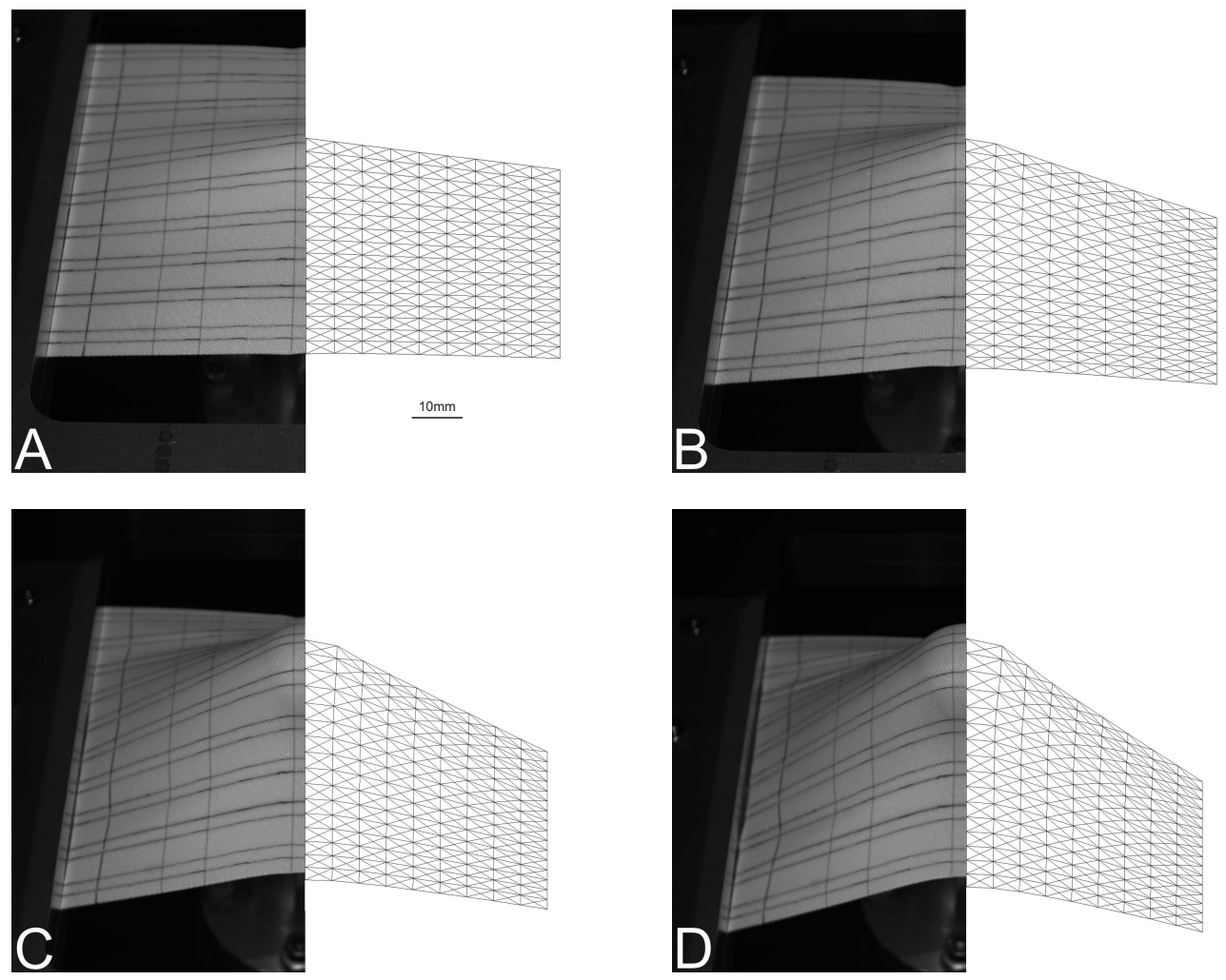

Figure 15: Comparisons of half of the deformed electronic textile during the punch test as obtained from the experiments (left) and the deformed model as predicted by the simulation (right). The four comparisons show the electronic textile at punch displacements of $10 \mathrm{~mm}$ (A), $20 \mathrm{~mm}$ (B), $30 \mathrm{~mm}$ (C) and $40 \mathrm{~mm}$ (D). Note that the images made during the experiment are truly three-dimensional while the deformations computed by the simulation only give an indication of the three-dimensional shape. (This can be observed by the left fixed edge in the experimentally obtained images that is oriented at an angle with respect to the vertical axis, while the right fixed edge in the simulation results is oriented exactly along the vertical axis.)

correspond rather well (based on Fig. 15) until a displacement of approximately $25 \mathrm{~mm}$ is reached. For larger displacements, the macroscopic shear angles deviate.

\subsection{Failure of the conductive wires}

The strains that occur during the punch experiment cannot directly be determined from the experiments. The damage of the conductive wires how- 
ever, can be investigated after the punch experiment. This gives a qualitative idea of the maximum strains that have occurred during the punch test in warp direction. To visualize the damage of the conductive wires, the same $\mathrm{X}$-ray equipment is used as for the warp tensile experiments in section 2.3. The damage at six locations indicated in Fig. 16 is shown in Fig. 17. One must take into account however, that the conductive wires in Fig. 5 have undergone uniaxial tension while the wires shown in this section have been subjected to more complex loading situations.

The engineering warp strains computed by the model at the six locations are also shown in Fig. 17. The warp strains are shown for a punch displacement of $52.5 \mathrm{~mm}$, because at this punch displacement the experimental curves decrease on average. Slip from the clamps has taken place at this displacement, but since this is difficult to asses, it is assumed that most samples have been exposed to this punch displacement.

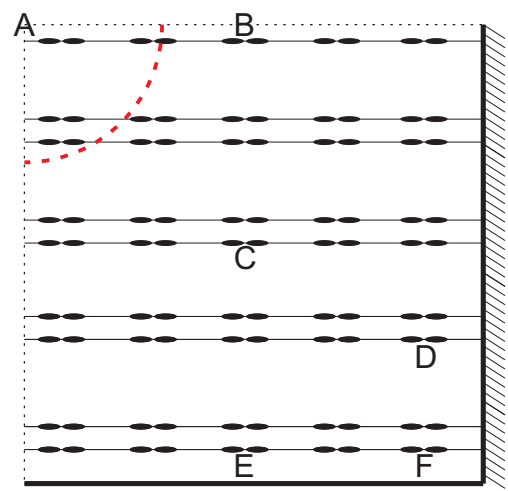

Figure 16: Schematic representation of the bottom left quarter of a sample in the punch test. The red dashed curve represents a quarter of the punch and the horizontal lines with small ellipsoids represent the conductive wires. Six regions are indicated by A to F, at which the residual deformations of the conductive wires after the punch experiment have been visualized using X-ray imaging (see Fig. 17).

At locations A and B it is clearly visible in Fig. 17 that several conductive wires have failed. Since in section 2.3 it has been established that failure of the wires starts at warp strains of $7 \%$, significantly larger strains have occurred at these locations. This corresponds with the large engineering warp strains that are observed in the simulation results at these locations (also indicated in Fig. 17). 

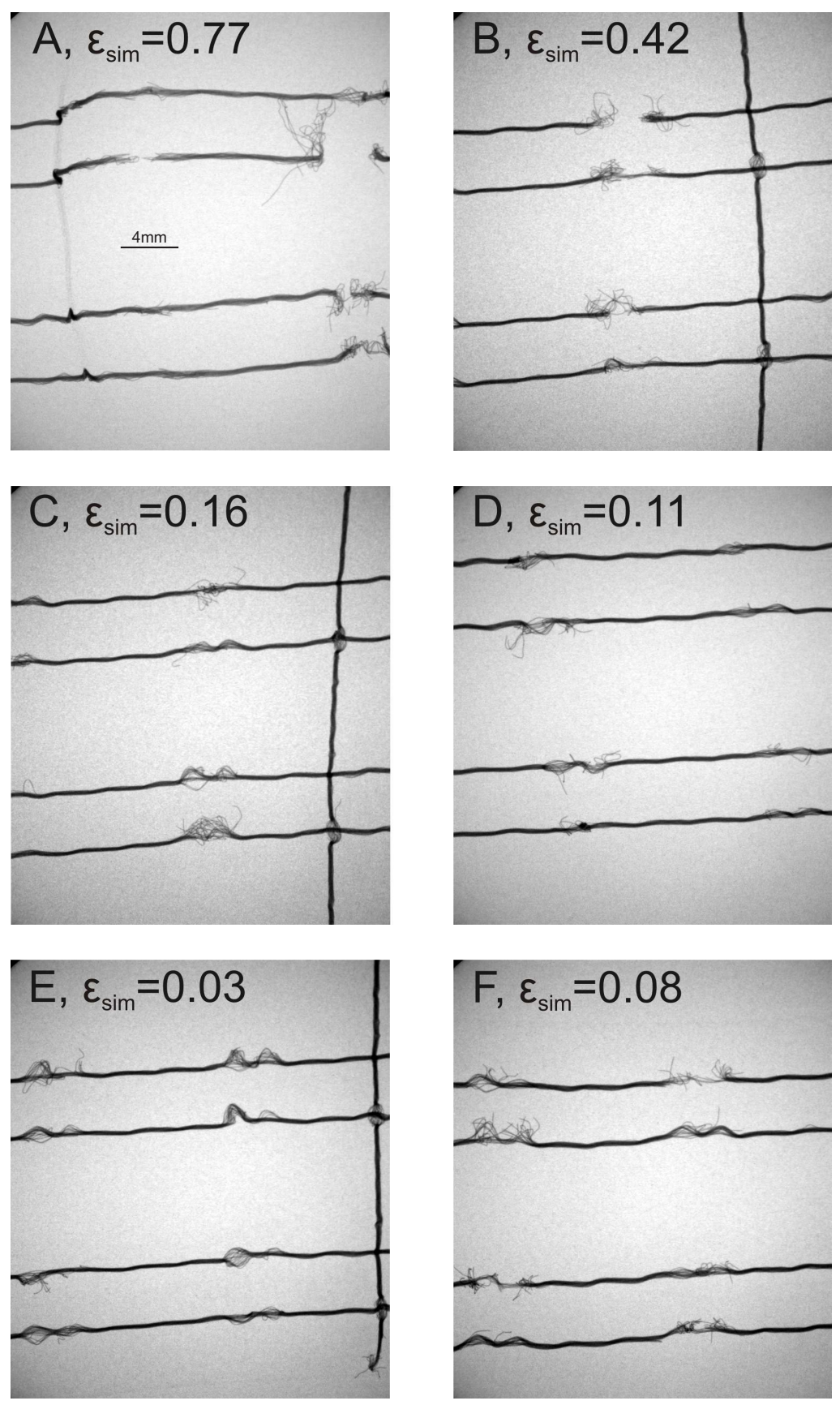

Figure 17: X-ray images of the electronic textile after the punch test. The damage of the conductive wires is shown for the locations A-F in Fig. 16. The engineering warp strains as computed in the simulation at a punch displacement of $52.5 \mathrm{~mm}, \epsilon_{\text {sim }}$, are also shown. 
At location $\mathrm{E}$ no failure can be seen but only plastic deformation of the conductive wires. The engineering warp strain of $3 \%$ computed at this location corresponds with this observation since it is significantly smaller than the warp strain of $7 \%$ at which failure initiates.

At locations C, D and F, it is visible that a substantial amount of plastic deformation has occurred in the wires (see again Fig. 17). The amount of damage is less than at locations A and B but clearly a substantial number of fibrils within several conductive wires have failed. This is in correspondence with the predictions since all predicted engineering warp strains are above the threshold of $7 \%$. The relatively large amount of damage at location $\mathrm{F}$ compared to locations $\mathrm{C}$ and $\mathrm{D}$ is quantitatively not completely in agreement with the predictions, since at location $\mathrm{F}$ a strain of $8 \%$ is predicted and the predicted strains at locations $\mathrm{C}$ and $\mathrm{D}$ are larger. However, qualitatively the model predicts failure correctly, since all predicted strains are larger than $7 \%$ at the locations where failure occurs.

\section{Conclusion}

The aim of this article was to present a straightforward experimental identification procedure for an in-plane lattice model of woven fabrics. The advantage of the presented identification approach is that the tensile responses in three in-plane directions can be directly used to separately determine the parameters of the three families of discrete elements in the lattice model. This has been established by ensuring that only the family of elements that are oriented in the loading direction during one of the three tensile tests contribute to the mechanical response. Therefore, no mutual influence of the different elements occurs during each tensile test and no (complex) inverse problem needs to be solved. The unit cell of the proposed lattice model is based on the mesoscopic structure of the textile, so that each conductive wire can be incorporated in the model if desired.

To ensure that a separate identification of the families of discrete elements is allowed two conditions must hold. First, the compressive response of the elements in the lattice model must be negligible compared to the tensile response of the elements. Second, the in-plane shear stiffness of the woven material must be compliant compared to the responses in the two principal in-plane directions. Since the latter generally holds for most woven materials, the lattice model and its identification procedure can be used more generally 
than for the electronic textile considered here. A disadvantage of these two conditions is that the lattice model cannot be used for cyclic loading.

The lattice model and its identification procedure are validated by an out-of-plane punch test on electronic textile, in which copper wires are incorporated to provide conductivity. In the punch test local strains of $55 \%$ occur in the most critical direction (in the direction of the conductive wires), so it can be considered as a stringent validation test. The results show that failure of the conductive wires is qualitatively, and to some extent quantitatively, well predicted by the lattice model; at all locations at which failure occurs in the experiments, strains larger than the failure strain of the conductive wires are predicted.

Furthermore, comparing the experimental data with the numerical results shows that the lattice model is accurate for small and moderate strains. For large biaxial deformation the predicted response of the lattice model is stiffer than the actual response of the fabric. The cause of this is that during large biaxial deformation, the diagonal elements, used only to describe the shear response, influence the responses in the two principal directions as a result of their extensive elongation. An alternative may therefore be to use rotational springs instead of truss elements to describe the in-plane shear response, which is not investigated in this paper. Future research will show the capabilities of such models.

\section{Acknowledgements}

The authors gratefully acknowledge Steven Luitjens and Koen van Os of Royal Philips Electronics for their enthusiastic input and the electronic textile provided by them and the partners involved in the PLACE-it project. This research is supported by the Dutch Technology Foundation STW, which is the applied science division of NWO, and the Technology Programme of the Ministry of Economic Affairs under Project Nr. 10104.

\section{References}

Apedo, K.L., Ronel, S., Jacquelin, E., Bennani, A., Massenzio, M., 2010, Nonlinear finite element analysis of inflatable beams made from orthotropic woven fabric, International Journal of Solids and Structures, Vol. 47, 20172033. 
Badel, B., Vidal-Sallé, E., Boisse, P., 2007, Computational determination of in-plane shear mechanical behaviour of textile composite reinforcements, Computational Materials Science, Vol. 40, 439-448.

Beex, L.A.A., Peerlings, R.H.J., Geers, M.G.D., 2011, A quasicontinuuum methodology for multiscale analyses of discrete microstructural models, International Journal for Numerical Methods in Engineering, Vol. 87, 701718.

Boisse, P., Gasser, A., Hivet, G., 2001, Analyses of fabric tensile behaviour: determination of the biaxial tension-strain surfaces and their use in forming simulations, Composites: Part A, Vol. 32, 1395-1414.

Boisse, P., Zouari, B., Daniel, J.L., 2006, Importance of in-plane shear rigidity in finite element analyses of woven fabric composite preforming, Composites: Part A, Vol. 37, 2201-2212.

Ben Boubaker, B., Haussy, B., Ganghoffer, J.F., 2007, Discrete models of woven structures. Macroscopic approach, Composites: Part B, Vol. 38, 498-505.

Dalton, A.B., Colins, S., Muñoz, E., Razal, J.M., Von Howard, E., Ferraris, J.P., Coleman, J.N., Kim, B.G., Baughman, R.H., 2003, Super-tough carbon-nanotube fibres, Nature, Vol. 423, 703.

Edmison, J., Jones, M., Nakad, Z., Martin, T., 2002, Using piezoelectric materials for wearable electronic textiles, Proceedings of the 6th International Symposium on Wearable Computers, 41-48.

Ha-Minh, C., Kanit, T., Boussu, F., Imad, A., 2011, Numerical multi-scale modeling for textile woven fabric against ballistic impact, Computational Materials Science, Vol. 50, 2172-2184.

Hamila, N., Boisse, P., 2008, Simulations of textile composite reinforcement draping using a new semi-discrete three node finite element, Composites: Part B, Vol. 39, 999-1010.

Han, D., Tsai, S.W., 2011, Interlocked composite grids design and manufacturing, Journal of Composite Materials, Vol. 37, 287-316. 
Kato, S., Minami, H., Yoshino, T., Namita, T., 1997, Analysis of membrane structures based on fabric lattice model considering viscous characteristics, Proceedings of the IASS international symposium on shell \& spatial structures, Singapore.

Kawabata, S., Niwa, M., Kawai, H., 1973, The finite-deformation theory of plain-weave fabrics. Part II: the uniaxial-deformation theory, Journal of the Textile Institute, Vol 64, 47-61.

King, M.J., Jearanaisilawong, P., Socrate, S., 2005, A continuum constitutive model for the mechanical behavior of woven fabrics, International Journal of Solids and Structures, Vol. 42, 3867-3896.

Liu, J.X., Chen, Z.T., Li, K.C., 2010, A 2D lattice model for simulating the failure of paper, Theoretical and Applied Fracture Mechanics, Vol. 54, $1-10$.

Lomov, S.V., Verpoest, I., 2006, Model of shear of woven fabrics and parametric description of shear resistance of glass woven reinforcements, Composites Science and Technology, Vol. 66, 919-933.

Lomov, S.V., Ivanov, D.S., Verpoest, I., Zako, M., Kurashiki, T., Nakai, H., Hirosawa, S., 2007, Meso-FE modelling of textile composites: Road map, data flow and algorithms, Composites Science and Technology, Vol. 67, 1870-1891.

Lomov, S.V., Boisse, Ph., Deluycker, E., Morestin, F., Vanclooster, K., Vandepitte, D., Verpoest, I., Willems, A., 2008, Full-field strain measurements in textile deformability studies, Composites: Part A, Vol. 39, 1232-1244.

Marlescu, D., Marlescu, R., Zamora, N.H., Stanley-Marbell, P., Khosla, P.K., Park, S., Jayaraman, S., Jung, S., Lauterbach, C., Weber, W., Kirstein, T., Cottet, D., Grzyb, J., Tröster, G., Jones, M., Martin, T., Nakad, Z., 2003, Electronic textiles: a platform for pervasive computing, Proceedings of the IEEE, Vol. 91, 1995-2018.

Nilakantan, G., Keefe, M., Bogetti, T.A., Adkinson, R., Gillespie, J.W.Jr., 2010, On the finite element analysis of woven fabric impact using multiscale modeling techniques, International Journal of Solids and Structures, Vol. 47, 2300-2315. 
Ostoja-Starzewski, M., 2002, Lattice models in micromechanics, Applied Mechanics Reviews, Vol. 55, 35-60.

Peng, X.Q., Cao, J., 2005, A continuum mechanics-based non-orthogonal constitutive model for woven composite fabrics, Composites: Part A, Vol. $36,859-874$.

Potluri, P., Sagar, T.V., 2008, Compaction modelling of textile preforms for composite structures, Composite Structures, Vol. 86, 177-185.

Sharma, S.B., Sutcliffe, M.P.F., Chang, S.H., 2003, Characterization of material properties for draping of dry woven composite material, Composites: Part A, Vol. 34, 1167-1175.

Sharma, S.B., Sutcliffe, M.P.F., 2004, A simplified finite element model for draping of woven material, Composites: Part A, Vol. 35, 637-643.

Ten Thije, R.H.W., Akkerman, R., 2008, Solutions to intra-ply shear locking in finite element analyses of fibre reinforced materials, Composites: Part A, Vol. 39, 1167-1176.

Verberne, C.W., 2011, A structural lattice model for electronic textile, Master's thesis (MT 11.19). Eindhoven University of Technology, Eindhoven, The Netherlands. 